\title{
Synthesis of Nonspherical Colloidal Particles with Anisotropic Properties
}

\author{
Jin-Woong Kim, ${ }^{\dagger,+}$ Ryan J. Larsen, ${ }^{\dagger}$ David A. Weitz $* \dot{\dagger}, \xi$
}

Division of Engineering and Applied Sciences, Harvard University, Cambridge, MA 02138,USA, Amore-Pacific Co. R\&D Center, 314-1, Bora-dong, Giheung-gu, Yongin-si, Gyeonggi-Do, 446-729, Korea, and Department of Physics, Harvard University, Cambridge, MA 02138,USA. E-mail: weitz@deas.harvard.edu

\section{More examples of anisotropic nonspherical particles}
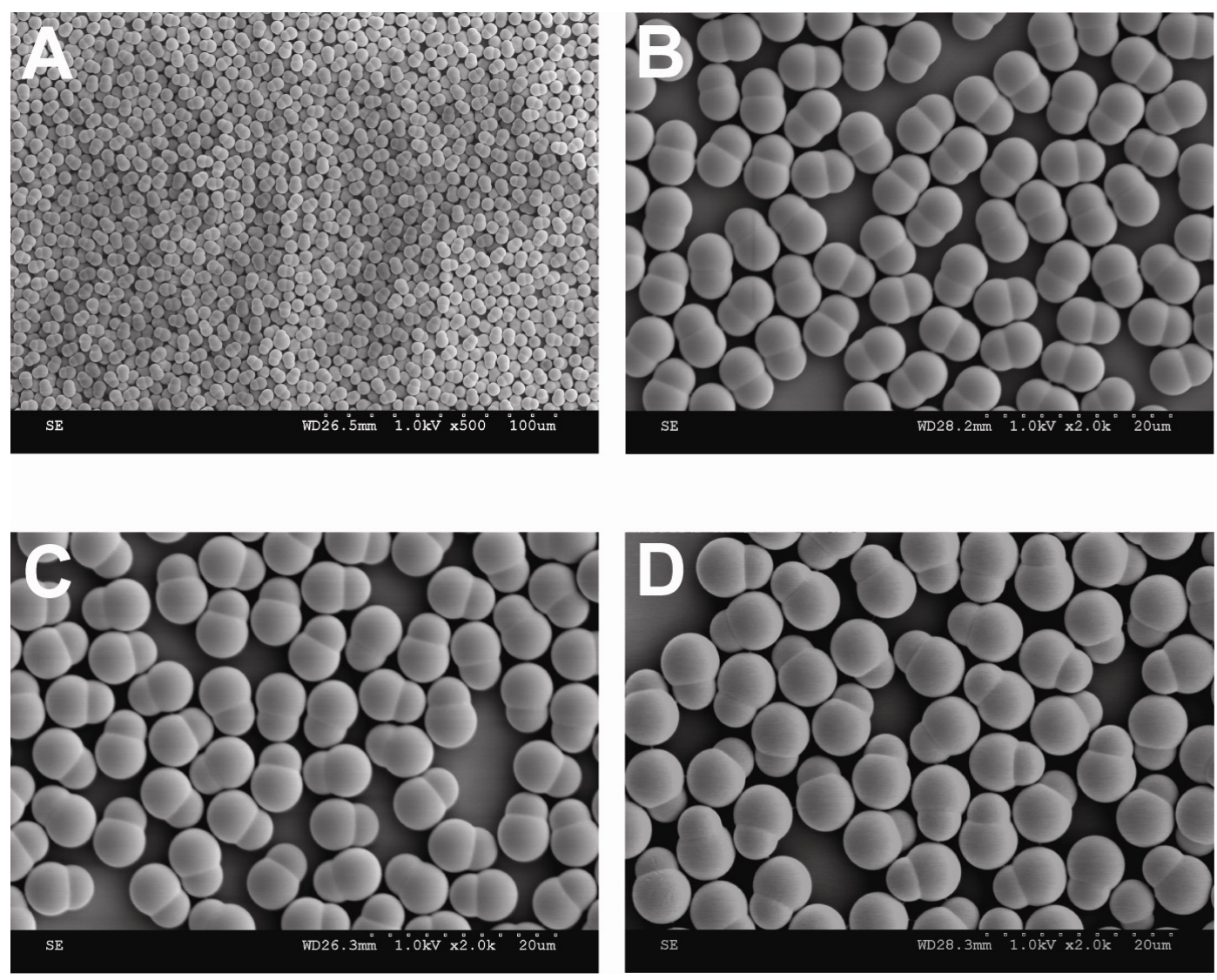

Figure S1. SEM micrographs of nonspherical PS particles. (A) PS dumbbell particles (CPS/styrene = 1/3.6, $\mathrm{v} / \mathrm{v}$ ) at low magnification. This pictures shows that the particles are very uniform in size and shape. PS dumbbell particles prepared by changing the swelling ratio of monomers to CPS particles; (B) CPS/styrene $=1 / 2.7(\mathrm{v} / \mathrm{v})$, (C) CPS/styrene $=1 / 3.6(\mathrm{v} / \mathrm{v})$, and (D) CPS $/$ styrene $=1 / 4.5(\mathrm{v} / \mathrm{v})$. 

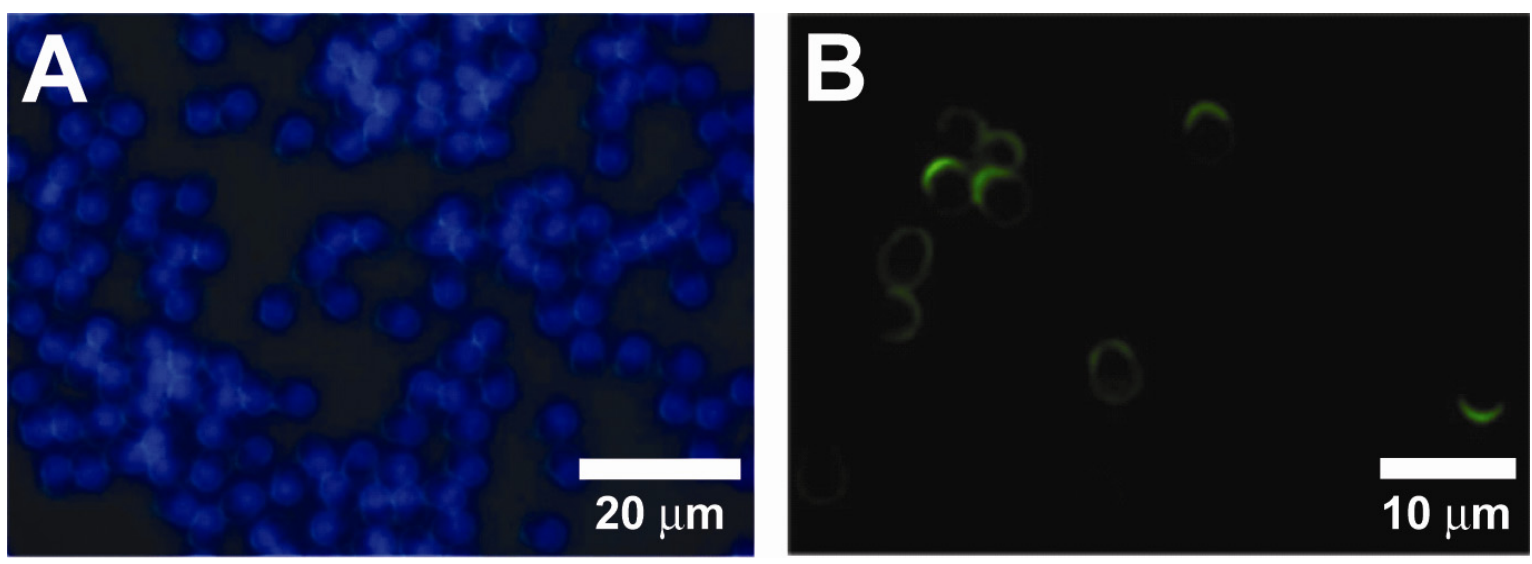

Figure S2. (A) A fluorescence microscope image of PS/PMMA dumbbell particles. To incorporate polymerizable dyes into one of the bulbs, we copolymerized 9-vinylanthracene $(\sim 0.25 \mathrm{wt} \%)$ to the CPS particles. The fluorescence images show that one of the bulbs, the CPS bulb is selectively dyed with a blue color. (B) A fluorescence microscope image of amphiphilic PS/PMMA dumbbell particles (CPS/MMA = 1/2, v/v). To synthesize these particles, we first copolymerized 5 vol\% 3-(trimethoxysilyl)-propyl acrylate to the CPS particles. Then, the CPS particles were swollen with MMA and ethylene glycol dimethacrylate (1 vol\%). The swollen monomers were polymerized at $70^{\circ} \mathrm{C}$ for $8 \mathrm{~h}$. After the synthesis of dumbbell particles, we reacted N-[3(trimethoxysilyl)propyl]-ethylene diamine with the silane groups on the surface of bulb a, the CPS part. Finally, the amine groups were labeled by reacting with fluorescein isothiocyanate. The fluorescence image shows that the fluorescein dyes are located richly on one of the bulbs.

\section{Information about movies}

Movie S1. The phase separation of monomer-swollen CPS particles. The CPS particles were swollen by styrene and DVB (1 vol\%). In this case, the monomer-swollen CPS particles didn't contain initiator (V-65B). Then, they were completely sealed in flat glass capillaries (inner diameters: $100 \mu \mathrm{m}$, VitroCom) and placed on a temperature-controlled hot stage. The temperature was $70 \pm 0.1{ }^{\circ} \mathrm{C}$. The phase separation was imaged with a digital camera every $3 \mathrm{sec}$ for $15 \mathrm{~min}$.

Movie S2. The phase separation of monomer-swollen CPS particles. The CPS particles were swollen by styrene, DVB ( $1 \mathrm{vol} \%)$, and V-65B $(0.5 \mathrm{wt} \%)$. To observe the effect of the polymerization on the phase separation kinetics, we prepared the monomer-swollen spherical CPS particles with $0.5 \mathrm{wt} \% \mathrm{~V}-65 \mathrm{~B}$ inside. Then, they were sealed completely in flat glass capillaries (inner diameters: $100 \mu \mathrm{m}$, VitroCom) and placed on a temperaturecontrolled hot stage. The temperature was $70 \pm 0.1{ }^{\circ} \mathrm{C}$. The phase separation was imaged with a digital camera every $3 \mathrm{sec}$ for $15 \mathrm{~min}$. 